\title{
Targeted treatment of psoriasis with adalimumab: a critical appraisal based on a systematic review of the literature
}

This article was published in the following Dove Press journal:

Biologics: Targets \& Therapy

10 June 2009

Number of times this article has been viewed

\author{
Jochen Schmitt \\ Gottfried Wozel \\ Department of Dermatology, Medical \\ Faculty Carl Gustav Carus, Technische \\ Universität Dresden, Germany
}

Correspondence: Jochen Schmitt Department of Dermatology, University Hospital Carl Gustav Carus, Technische Universität Dresden, Fetscherstr. 74, D-0I 307 Dresden, Germany

Tel +49 35I-458242I

Fax +49 35I-4585326

Email jochen.schmitt@uniklinikumdresden.de

\begin{abstract}
Psoriasis is a chronic inflammatory disease affecting $2 \%$ to $3 \%$ of the population in Western countries. Psoriasis is associated with limited quality of life, cardiovascular disease, and depression. The approval of injectable biological agents has revolutionized the management of moderate to severe psoriasis. Adalimumab is a human monoclonal antibody against tumor necrosis factor (TNF) alpha approved for moderate-to-severe plaque-type psoriasis and psoriatic arthritis (PsA). This systematic review summarizes the evidence concerning the efficacy, clinical effectiveness, safety, and cost-effectiveness of adalimumab in the treatment of psoriasis. Five randomized controlled trials demonstrated the efficacy of adalimumab in moderate-to-severe plaque-type psoriasis and PsA with PASI-75 response rates of 53\% to $80 \%$ and ACR-20 response rates of $39 \%$ to $58 \%$ after 12 to 16 weeks of treatment. In clinical practice patients who have not responded to one TNF antagonist may respond to another TNF antagonist. Adalimumab has similar or better cost-effectiveness than other biologics, but is less efficient than methotrexate and cyclosporine. Adalimumab is generally well tolerated. Patients should be evaluated for active/latent tuberculosis, serious infections, and other contraindications prior to initiation of adalimumab therapy. Future studies should investigate the comparative efficacy of adalimumab and other biologic and prebiologic agents. Recently established registries will yield additional data on the effectiveness and long-term safety of adalimumab.
\end{abstract}

Keywords: adalimumab, biologic, efficacy, effectiveness, efficiency, psoriasis, treatment, safety

\section{Introduction}

Psoriasis is an immune-mediated chronic inflammatory disease affecting approximately $2 \%$ to $3 \%$ of the population in Western countries. ${ }^{1}$ Among several clinical phenotypes chronic plaque-psoriasis is most frequent and accounts for about $90 \%$ of cases. ${ }^{1,2}$ Psoriasis may cause substantial problems in everyday life. ${ }^{3}$ Several studies suggested that patients with severe psoriasis have an increased risk for cardiovascular disease, depressive illness, and a decreased life expectancy. ${ }^{4-9}$ Currently incurable, psoriasis causes remarkable direct costs, work limitations and productivity loss. ${ }^{2,10-12}$

Systemic treatments are limited to patients with moderate-to-severe psoriasis in whom the activity of skin lesions and concurrent symptoms cannot be sufficiently controlled with topical treatments and with phototherapy. ${ }^{13-15}$

The history of treatment of psoriasis in past decades reflects uncertainty especially in terms of the pathogenesis of the disease. For example, the presence of large granulocyte accumulation and evidence of increased $\mathrm{LTB}_{4}$ in involved skin, discovered in the 1980s, and later hyperexpression of interleukin IL-8 suggested at that time that both mediators could play a crucial role in psoriasis (so-called 'neutrophilic dermatosis'). 
However, clinical trials with $\mathrm{LTB}_{4}$ or IL-8 antagonists were disappointing and showed no relevant antipsoriatic effects. In the mid 1980s intense investigation on cytokine production by T-cells led to the development of the T-helper 1/T-helper 2 paradigm. ${ }^{16}$ The shift from psoriasis as neutrophilic dermatosis to 'T-cell-mediated disease' finally came, among others, from the convincing clinical results of ciclosporine. In addition, a substantial body of experimental evidence demonstrated that indeed the T-cell has to be seen as a key effector cell type in psoriatic inflammation (eg, SCID mice experiments). Parallel advances in research converged on proinflammatory cytokine tumor necrosis factor alpha (TNF $\alpha$ ). Our knowledge about TNF $\alpha$ as a key cytokine has led to therapies targeting selective TNF $\alpha$ in psoriasis and other immune-mediated inflammatory diseases, in which lesional and systemically elevated levels of TNF $\alpha$ have been found. In this line rapid and substantial improvement of psoriasis and PsA by TNF $\alpha$ antagonists has been shown. On the other hand CD4+ T cells have been subdivided into different subsets, largely on the basis of the cytokines they generate. Such subsets include Th1, Th2, and regulatory $\mathrm{T}$ cells (Tregs). Recently another population of $\mathrm{T}$ cells has been described that is characterized by production of IL-17. ${ }^{17}$ In this context the heterodimeric IL-12/IL-23 have been found to play a major role in determining the differentiation of Th1 and Th 17 cells. Thus, targeting IL-12/23 p40 has led to a novel therapeutic approach as evidenced in numerous clinical trials in patients with psoriasis by use of monoclonal antibodies like ustekinumab or ABT874. ${ }^{18-20}$ In summary, in the past 15 years much progress has been made in new exciting treatment modalities for psoriasis and PsA.

Today, three TNF $\alpha$ antagonists are registered for treating psoriasis and PsA (etanercept, infliximab, adalimumab). A novel human TNF $\alpha$ monoclonal antibody is expected to be approved for psoriatic arthritis in the near future (golimumab).

According to the $\mathrm{T}$ cell inactivating monoclonal anti- $\beta 2$ integrin lymphocyte function-associated antigen-1 antibody efalizumab, recently the EMEA's Agencies Committee for Medicinal Products for Human Use (CHMP) has reviewed all available data on safety and effectiveness of efalizumab and recommended in a press release on February 19, 2009 that the marketing authorization for the drug should be suspended in the EU. ${ }^{21}$ One important issue for this decision was the occurrence of progressive multifocal leucencephalopathy (PML) in patients treated with efalizumab. ${ }^{21}$ As alefacept another $\mathrm{T}$ cell inhibitor - is not approved in EU (except Switzerland), there is as yet no biologic $\mathrm{T}$ cell inhibitor available for treating psoriasis. Therefore, TNF $\alpha$ inhibitors are currently the most important drugs in the therapeutic management of psoriasis and PsA. Because of the therapeutic importance of the family of TNF $\alpha$ antagonists among several medical disciplines such as rheumatology, gastroenterology, and dermatology, we intend to give a critical review about clinical efficacy and safety of adalimumab as a fully human monoclonal IgG1 TNF $\alpha$ antibody, especially in psoriasis.

\section{TNF $\alpha$}

TNF $\alpha$ belongs to the TNF $\alpha$ super family that constitutes at least 20 proteins. ${ }^{22}$ According to the revised nomenclature proposed by Schottelius et al TNF $\alpha$ is denoted by the acronym TNFSF 2 (tumor necrosis factor super family number 2 ). ${ }^{22}$ From a clinical perspective, $\mathrm{TNF} \alpha$ is a general term that includes soluble TNF $\alpha(\mathrm{sTNF} \alpha)$ and transmembrane $\mathrm{TNF} \alpha$ $(\operatorname{tm} \mathrm{TNF} \alpha)$.

At low concentrations in tissues TNF $\alpha$ is thought to have beneficial effects such as prevention of infections. At high concentrations TNF $\alpha$ can lead to excess inflammation and organ injury. In a number of diseases $\mathrm{TNF} \alpha$ is generally considered to be a proinflammatory cytokine. Activities ascribed to TNF $\alpha$ in psoriasis are described in detail elsewhere. ${ }^{23-34}$

Newly synthesized pro-TNF $\alpha$ is expressed on the plasma membrane as a $26 \mathrm{kDa}$ protein and is thereafter cleaved in the extracellular domain by the TNF $\alpha$-converting enzyme (TACE, syn. ADAM 17) to release a mature soluble $17 \mathrm{kDa}$ protein as monomer. ${ }^{35}$ For biological activity trimerization is required, so that the generation of TNF $\alpha$ homotrimers with $51 \mathrm{kDa}$ is realized by self-aggregation. The biological response of TNF $\alpha$ is mediated through two structurally distinct receptors:

Type 1 (TNFR1, syn.: p60, p55, CD 120a) and

Type 2 (TNFR2, syn.: p80, p75, CD 120b). ${ }^{35}$

Both receptors represent transmembrane glykoproteins with cysteine-rich repeats in the extracellular $\mathrm{N}$-terminal domain. ${ }^{36}$ Nearly all nucleated cells express TNF $\alpha$ receptors, whereas their distribution varies with cell type. The receptors can be cleaved from the cell surface by enzymes of the matrix metalloproteinase family in response to inflammatory signals. The general biological effects of the major proinflammatory cytokine TNF $\alpha$ exhibit a wide diversity of functional activities. ${ }^{22,36,37}$

The significance of TNF $\alpha$ especially in psoriasis and PsA has been shown in numerous experimental and clinical investigations. $^{22}$

1. Increased levels of TNF $\alpha$ in lesional psoriatic skin;

2. Increased TNF $\alpha$ mRNA expression in peripheral blood mononuclear cells of psoriatic patients; 
3. Increased plasma concentration levels of TNF $\alpha$ as bioactive trimeric molecule in psoriatic patients;

4. Differential expression of TNFR 1 and TNFR 2 in normal, uninvolved, and lesional skin of psoriasis patients.

However, from the clinical perspective the most impressive issue is that neutralization of TNF $\alpha$ in psoriasis by TNF $\alpha$ antagonists results in a high grade of amelioration of disease activity. Thus, clinical trials and practice have, in turn, strongly implicated $\mathrm{TNF} \alpha$ in the pathogenesis of psoriasis and PsA..$^{38}$

\section{Adalimumab: structure and mechanism}

Adalimumab, firstly described in the literature under the laboratory name D2E7, is a recombinant human immunoglobulin (IgG1) monoclonal antibody containing only human peptide sequences. Originally it was developed by BASF Pharma (Ludwigshafen Germany) to create a fully human monoclonal antibody with the goal of decreasing the antigenicity in order to achieve a greater therapeutic potential. ${ }^{39}$

Adalimumab is indistinguishable in structure and function from naturally occurring human IgG1. It is produced by phage display technology and consists of 1330 amino acids with a molecular weight of approximately $148 \mathrm{kDa}$. The terminal half-life is about 14 days. ${ }^{40-43}$ Relevant pharmacokinetic parameters of adalimumab are summarized in Table 1.

Adalimumab has a very high specificity and affinity for $\mathrm{TNF} \alpha$. It does not bind to or inactivate lymphotoxin $\alpha$ (TNF $\beta)$. The antibody binds to soluble and membrane bound TNF $\alpha$ in a dose-dependent manner before TNF $\alpha$ interacts with the p55 and p75 cell surface TNF $\alpha$ receptors. By preventing the binding of TNF $\alpha$ to its specific receptors, adalimumab blocks

Table I Pharmacokinetic properties of adalimumab ${ }^{40-43}$

\begin{tabular}{ll}
\hline Parameter & Value \\
\hline Absolute bioavailability & $64 \%$ \\
Time to maximum concentration & $|3| \pm 56 \mathrm{hours}$ \\
Maximum serum concentration & $4.7 \pm 1.6 \mu \mathrm{g} / \mathrm{mL}$ \\
Volume of distribution & 4.7 to $6.0 \mathrm{~L}$ \\
Steady-state through concentration & $\sim 5 \mathrm{mg} / \mathrm{mL}$ \\
Total body clearance & $0.18-0.27 \mathrm{ml} / \mathrm{min}$ \\
Terminal half-life & $\sim 14$ days \\
Time to & \\
Initial response & $\mathrm{I}-7$ days \\
Peak response & Within 3 months \\
\hline
\end{tabular}

the proinflammatory activity of the cytokine. The ability of adalimumab to neutralize TNF $\alpha$ bioactivity was demonstrated in different in vitro cell systems. In vitro, adalimumab lyses surface TNF $\alpha$-expressing cells in the presence of complement. Molecular analyses reveal that adalimumab forms stable trimer complexes with TNF $\alpha$ consisting of alternating three adalimumab and three TNF $\alpha$ molecules with the molecular weight of $598 \mathrm{kDa}^{44}$ It has been shown that adalimumab modulates responses that are induced or regulated by $\mathrm{TNF} \alpha$, including expression of adhesion molecules, serum concentrations of cytokines, matrix metalloproteinases, reactive oxygen species of polymorphonuclear neutrophils, and dendritic cells in psoriatic plaques ${ }^{41}$ Adalimumab has no effect on skin UV response in patients suffering from rheumatoid arthritis ${ }^{45}$ In summary, these findings of characteristic properties of adalimumab all are in line with preclinical demands of a therapeutically useful TNF $\alpha$ antagonist. Nonclinical toxicological assessment of adalimumab included studies in several animal species (eg, mice, rat, cynomolgus monkeys). These studies demonstrated a safe toxicological profile providing a significant safety above dose levels investigated in clinical trials. Moreover, adalimumab did not show any signals for inducing mutagenicity in the Ames test or cause clastogenicity in vivo in mice..$^{40-43}$

\section{Methods}

We systematically reviewed all published articles reporting original data on the efficacy of adalimumab for psoriasis in the setting of randomized controlled trials (RCT), its clinical effectiveness in daily practice (with $\mathrm{n} \geq 30$ ), its efficiency/ cost-effectiveness, as well as its safety and tolerability as reported in RCTs and in selected case reports. Additionally, we reviewed all pharmaco-genetic studies assessing genetic predispositions for response to adalimumab treatment of psoriasis patients.

Relevant articles were identified searching Medline from its inception until January 2009 for combinations of the search terms "psoriasis" and "adalimumab". Animal studies were excluded. One investigator (JS) searched, reviewed, and abstracted data. Relevant data were abstracted using a standardized data abstraction form which was pilot tested for this systematic review. Primary measure of the efficacy of adalimumab in the treatment of plaque-type psoriasis was the proportion of patients with at least $75 \%$ reduction in psoriasis area severity index (PASI-75 response rate). ${ }^{46}$ The primary measure of the efficacy of adalimumab in the treatment of PsA was the proportion of patients with at least $20 \%$ improvement according to the American College of 
Rheumatology (ACR) criteria (ACR-20 response rate). ${ }^{47}$ Additionally, PASI-50 and PASI-90 response rates and ACR-50 and RCA-70 response rates are reported from RCTs investigating adalimumab in patients with plaque-type psoriasis and PsA, respectively. Patient-reported outcomes such as the effect of adalimumab therapy on health-related quality of life, illness-related stress, disability, depressive symptoms were also abstracted from RCTs. Evidence from effectiveness studies and from economic evaluations was summarized using the endpoints used in the different studies identified. Adverse events and withdrawals were summarized as mean monthly incidence rates.

No external sponsor was involved in this study. No persons other than the authors of this manuscript were involved in the design, analysis, and interpretation of data, in the writing of the report, and in the decision to submit the article for publication.

\section{Results}

We identified a total of 201 articles, 102 of which were reviews not reporting original data. From the remaining 99 papers we included

- 5 RCTs (14 papers $^{48-61}$ ) assessing the safety and efficacy of adalimumab for plaque-type psoriasis and/or PsA,

- 3 studies $^{62-64}$ investigating the clinical effectiveness of adalimumab for patients with psoriasis in daily practice,

- 2 pharmaco-genetic studies ${ }^{65,66}$ reporting an association of different gene-polymorphisms and response to adalimumab treatment for psoriasis,

- 3 economic evaluations ${ }^{67-69}$ looking at the cost-effectiveness of adalimumab treatment for psoriasis, and

- 16 case reports/case series ${ }^{70-85}$ discussing the safety of adalimumab and adverse events possibly related to adalimumab therapy administered for psoriasis.

\section{Efficacy of adalimumab in the treatment of plaque-type psoriasis and PsA}

Table 2 summarizes relevant data from the RCTs identified. All RCTs exclusively included adults with moderate-to-severe plaque-type psoriasis and/or PsA.

Three studies assessed the efficacy of adalimumab in moderate-to-severe plaque-type psoriasis. ${ }^{48-50}$ After 12 weeks of double-blind treatment with adalimumab $40 \mathrm{mg}$ and adalimumab $40 \mathrm{mg}$ every other week (eow), 80\% and $53 \%$ of patients showed at least $75 \%$ PASI improvement, respectively, whereas only $4 \%$ of the patients who had received placebo met the PASI-75 response criterion. ${ }^{48}$
Based on the intention-to-treat population, PASI-75 response rates the end of the open-label extension study until week 60 after treatment were $64 \%$ after treatment with adalimumab $40 \mathrm{mg}$ weekly and $56 \%$ in patients receiving adalimumab 40 mg eow..$^{48}$

In a phase III double-blind, placebo-controlled RCT including 1212 patients with moderate-to-severe plaque-type psoriasis, Menter et al reported PASI-75 response rates of $71 \%$ after 16 weeks and $70 \%$ after 24 weeks of treatment with adalimumab 40 mg eow. ${ }^{49}$ To investigate the loss of response within 19 weeks after discontinuation of adalimumab therapy, patients responding to adalimumab (ie, met the PASI-75 response criterion) in week 33 were re-allocated and continued double-blind treatment with adalimumab or were switched to placebo and treated accordingly for the following 19 weeks (until week 52). Loss of response, defined as $<$ PASI-50 and $\geq 6$ Point increase in PASI, was observed in $28 \%$ of patients switched to placebo and $5 \%$ of patients continuing adalimumab therapy. ${ }^{49}$ This indicates that continuous treatment with adalimumab is required in many patients to maintain sustained effectiveness and that adalimumab does not appear to modify the course of plaquetype psoriasis in a way that long-term remissions are to be expected, at least not in all patients.

The only head-to-head trial comparing adalimumab against another systemic treatment for moderate-to-severe psoriasis investigated the comparative efficacy of adalimumab $40 \mathrm{mg}$ eow $(\mathrm{n}=108)$ versus methotrexate (MTX; $7.5 \mathrm{mg}$ orally, increased as needed and as tolerated to $25 \mathrm{mg}$ weekly; $n=110)$ versus placebo $(n=53)$. After 16 weeks of double-blind treatment, $80 \%$ of adalimumab-treated patients achieved PASI-75, compared with $36 \%$ for methotrexate and 19\% for placebo PASI-50 and PASI-90. Response rates were also higher in patients receiving adalimumab than in those treated with MTX, so that the authors concluded that adalimumab demonstrated significantly superior efficacy in plaque-type psoriasis compared with MTX ${ }^{50}$ This conclusion, however, was criticized because the trial was designed as a noninferiority study, the demonstration of superiority of adalimumab over MTX being based on a post-hoc analysis. From a methodological perspective, it can therefore only be concluded that adalimumab is not worse than MTX, although the reported data suggest that it may be superior. ${ }^{55}$

Two double-blind, placebo-controlled trials were conducted to investigate the efficacy of adalimumab $40 \mathrm{mg}$ eow in patients with moderately to severely active PsA and a history of inadequate response to nonsteroidal anti-inflammatory drugs (Table 2). ${ }^{56-59}$ 


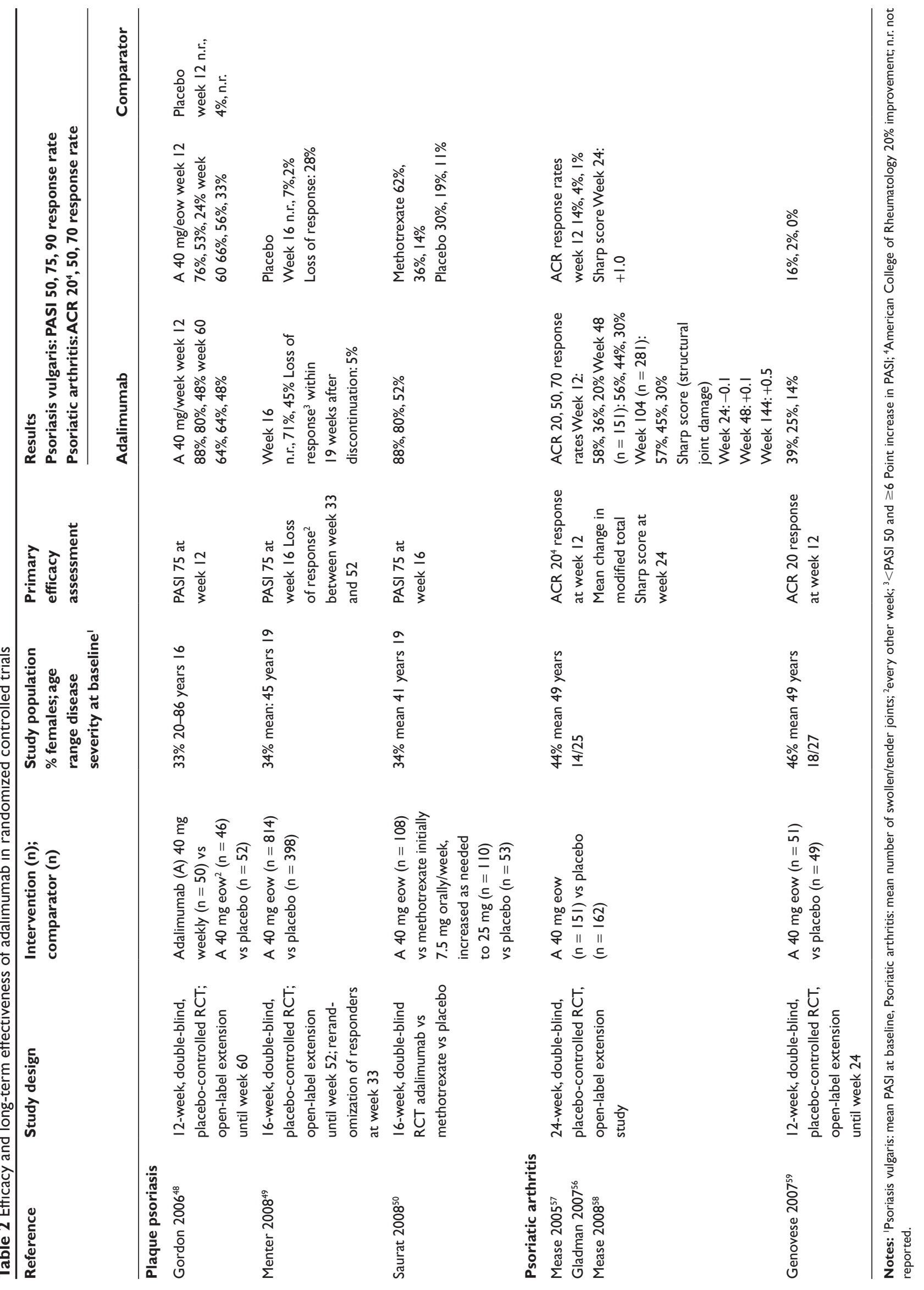


Genovese et al reported ACR-20 response rates of 39\% after treatment with adalimumab for 12 weeks versus $16 \%$ for placebo $(p=0.01)$. At week 24 , after an open-label extension period, $65 \%$ of patients met the ACR2 0 response criterion. ${ }^{59}$

Another study published by Mease et $\mathrm{al}^{57}$ demonstrated that adalimumab significantly improves joint manifestations as assessed by the ACR criteria and also inhibited structural changes on radiographs. At week 12, 58\% of the adalimumabtreated patients achieved an ACR-20 response, compared with $14 \%$ of the placebo-treated patients. Similar response rates were maintained in the adalimumab group until week $104 .^{58}$ At week 24, the mean change in the modified total Sharp score was -0.2 in patients receiving adalimumab and 1.0 in those receiving placebo indicating that adalimumab inhibits the progression of structural joint changes attributed to PsA. ${ }^{57}$

\section{Patient-reported outcomes}

Each randomized controlled trial identified also assessed a set of patient-reported outcomes as secondary endpoints, which were reported in detail in secondary publications. ${ }^{51-54,60,61}$ One secondary analysis utilizing data from an $\mathrm{RCT}^{49}$ indicated that clinical efficacy of adalimumab therapy translates into improved wellbeing and health-related quality of life as assessed by the Short Form-36 (SF-36) Health Survey ${ }^{86,87}$ scores which showed improvements both in physical and in mental health after 16 weeks of adalimumab therapy. ${ }^{51}$ The head-to-head study comparing adalimumab with MTX in patients with plaque-type psoriasis $^{50}$ indicated that adalimumab leads to an improved quality of life as assessed by means of the Dermatology Life Quality Index (DLQI) ${ }^{88}$ and the EuroQOL 5D (EQ-5D) ${ }^{89}$ and is also beneficial for disease symptoms such as pruritus and pain. ${ }^{52}$ It has also been shown that adalimumab therapy increases work productivity and activity ${ }^{53}$ which is particularly important in light of the known adverse effect of psoriasis on work productivity and high corresponding indirect cost. ${ }^{12}$

Pooled data from different trials totaling 1469 patients with moderate to severe plaque-type psoriasis indicated that the degree of PASI response correlates with the improvement of quality of life, PASI-90 or PASI-100 responders experiencing greater improvements in DLQI total score than PASI-75 responders. ${ }^{60}$

For patients with PsA it has been demonstrated that adalimumab therapy decreases functional impairment, health-related quality of life, fatigue, and pain. ${ }^{61}$

\section{Effectiveness in clinical practice}

Data on studies evaluating the clinical effectiveness of adalimumab for plaque-type psoriasis and/or for PsA in daily clinical practice are summarized in Table 3.
Papoutsaki et al assessed the effectiveness of adalimumab therapy in 30 patients affected by moderate-to-severe plaque-type psoriasis with or without PsA, who had failed to respond to, did not tolerate, or had contraindications against the other biologic and nonbiologic treatment options for psoriasis. ${ }^{62}$ Adalimumab $40 \mathrm{mg}$ once a week was administered in monotherapy for 24 weeks with assessment of PASI and Ritchie articular index (the latter only in the subset of patients with PsA) at week 12 and 24. PASI-75 response rates at week 12 and 24 were $87 \%$ and $83 \%$, respectively. At week 24 , arthritis had significantly improved from mean baseline Ritchie articular index 10 to $2 .{ }^{62}$ Although this study is limited by open-label treatment, the absence of a control group, and missing information on concurrent topical antipsoriatic treatment, it gives good evidence that adalimumab effectively controls plaque-type psoriasis and PsA in many patients refractory to other biologic and conventional agents.

Heiberg et al compared the effectiveness of adalimumab, infliximab, and etanercept in patients with PsA, rheumatoid arthritis, and ankylosing spondylitis by means of the 1-year retention rates of the different medications. ${ }^{63}$ This is a very interesting approach as retention rates are a good global indicator for effectiveness, patient satisfaction, and tolerability. The analyses comprised 172 anti-TNF $\alpha$ treatment courses in patients with PsA (adalimumab $\mathrm{n}=28$; infliximab $\mathrm{n}=48$; etanercept $\mathrm{n}=96$ ). Withdrawal rates were lowest for adalimumab (14.3\%) than for both other anti-TNF $\alpha$ agents approved at the time the study was conducted (infliximab $25.0 \%$; etanercept $24.0 \%$ ). The authors also calculated hazard ratios for treatment termination with adjustments for age, sex, investigator's global assessment, and concomitant MTX therapy. Concomitant MTX therapy was associated with lower withdrawal rates of anti-TNF $\alpha$ agents. This analysis, however, was not restricted to adalimumab, but also considered etanercept and infliximab. ${ }^{63}$

A retrospective chart review including 49 patients moderate-to-severe plaque-type psoriasis indicated that $88 \%$ and $78 \%$ of patients were considered as "clear" or "almost clear" according to physician's global assessment of disease severity after 3 and 12 months of treatment with adalimumab $40 \mathrm{mg}$ per week. According to this study, long-term effectiveness of adalimumab was independent of previous biological treatment. Response rates after 12 months of adalimumab therapy in the subgroup of patients previously treated with infliximab and/or etancercept was $78 \%$. Within the 12-month study period 5 patients (10\%) discontinued adalimumab because of lack of effectiveness. ${ }^{64}$ The main drawback of this study is that only patients observed for at least 


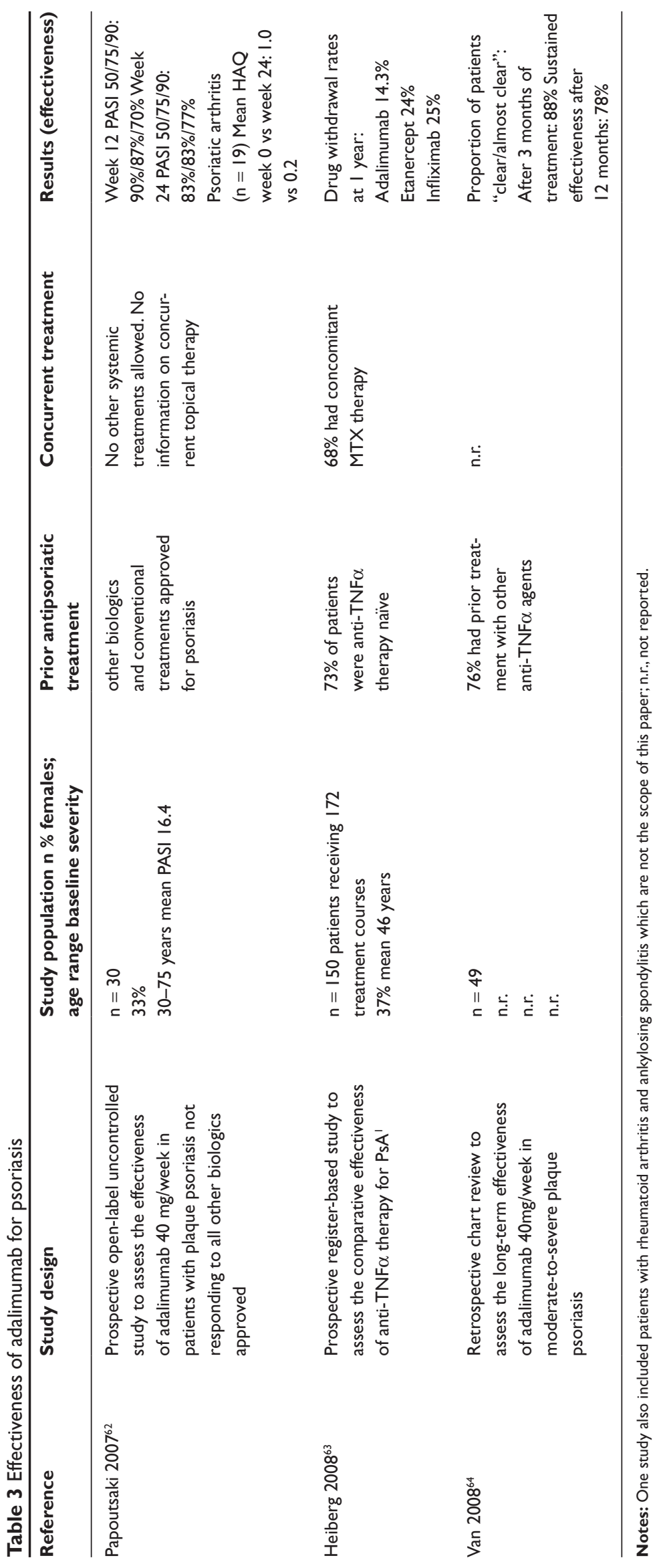


12 months were included, which may have caused selection bias with consecutive overestimation of effectiveness.

\section{Pharmaco-genetic studies}

We identified two pharmaco-genetic investigations evaluating the response to anti-TNF $\alpha$ agents including adalimumab with TNF $\alpha$-gene polymorphism and Fc $\gamma$-receptor polymorphism, respectively ${ }^{65,66}$ Based on a clinical sample of 30 patients with rheumatoid arthritis and 5 patients with PsA, Tutuncu et al reported that the response to anti-TNF $\alpha$ treatment varied with regard to the Fc $\gamma$-receptor receptor type IIIA-158 genotype, the low-affinity F/F homozygous genotype being significantly associated with response to TNF $\alpha$-inhibitor therapy $(\mathrm{p}<0.01) .{ }^{66}$ Seitz et al observed in another clinical sample of patients with PsA, rheumatoid arthritis, and ankylosing spondylitis treated with adalimumab $(\mathrm{n}=10)$, infliximab $(n=63)$, or etanercept $(n=13)$, respectively, that treatment response significantly differed with regard to the G-to-A polymorphism at position -308 in the promoter of the TNF $\alpha$-gene. All patients with the A/A genotype failed to respond to treatment, whereas good response was exclusively seen in patients with the G/G genotype. ${ }^{65}$ These studies are very promising and may help to better identify the subgroup of psoriasis patients with psoriasis benefiting most from adalimumab therapy. Replications of these promising findings and similar studies in patients with plaque-type psoriasis are necessary.

\section{Safety and tolerability}

Tables 3 and 4 summarize, respectively, safety data from clinical trials on adalimumab for psoriasis and case reports highlighting rare adverse reactions occurring in patients with psoriasis under adalimumab therapy, which may have been related to this agent.

The monthly incidence rate of injection site reaction ranged from $1.2 \%^{57}$ to $3.4 \%{ }^{48}$ in clinical trials (Table 4). Within 2 years of exposure to adalimumab 20/298 patients with PsA discontinued treatment because of adverse events. ${ }^{58}$ In this long-term study 3 patients (1.0\%) died within the observation period, 2 of whom had myocardial infarction that was classified as probably not related to adalimumab, and 1 experienced sudden death after acute pulmonary edema that was classified as possibly related to adalimumab. ${ }^{58}$ No deaths were observed in other clinical trials included in this systematic review. ${ }^{48-50,59}$ Interestingly, in the RCT by Genovese et $\mathrm{al}^{59}$ to assess the efficacy and safety of adalimumab for PsA, adverse events occurred significantly more frequently in patients receiving placebo than in those receiving adalimumab $(\mathrm{p}<0.01)$.
The specific side effects associated with anti-TNF therapy in general should be discussed at greater length. As discussed already, TNF $\alpha$ plays a central role in the inflammation and cellular immune response. TNF $\alpha$ is a cytokine of the innate immune system critical in the surveillance of malignancies and infections. Thus, anti-TNF agents, including adalimumab, may affect a patient's defence against infections and malignancies.

In the setting of clinical trials, upper respiratory tract infections and nasopharingitis were observed more frequently in patients treated with adalimumab than in those receiving placebo (Table 4).

One area that has been of particular concern with the advent of anti-TNF $\alpha$ biologic therapies has been the reactivation of latent tuberculosis $(\mathrm{Tb}){ }^{90,91} \mathrm{TNF} \alpha$ is an important cytokine in preventing $\mathrm{Tb}$ infection and in keeping latent $\mathrm{Tb}$ infection from becoming active disease. Following the National Psoriasis Foundation consensus statement on screening for latent $\mathrm{Tb}$ in patients with psoriasis treated with systemic and biologic agents, it is therefore of utmost importance to appropriately screen all patients for latent $\mathrm{Tb}$ infection prior to initiating any immunologic therapy. The tuberculin skin test is still used most frequently as a screening tool for latent $\mathrm{Tb}$. This strategy, however, is limited by the poor specificity of the tuberculin skin test in populations vaccinated with bacille Calmette-Guérin (BCG) and its low sensitivity in immunosuppressed persons. Two blood tests (T-SPOT. TB and QuantiFERON-TB Gold), based on detection of IFN- $\gamma$ released by $T$ cells in response to 2 unique antigens that are highly specific for Mycobacterium tuberculosis but absent from BCG vaccine and most nontuberculous, mycobacteria offer an improvement on the skin test. Both tests are not confounded by prior BCG vaccination and also have operational advantages over the skin test because no return visit is required, results are available by the next day, and repeated testing does not cause boosting. ${ }^{92,93}$

Delaying immunologic therapy until latent $\mathrm{Tb}$ infection prophylaxis is completed is preferable. ${ }^{94}$ In the RCTs included in this review, patients were screened for latent $\mathrm{Tb}$ prior to inclusion and not eligible if $\mathrm{Tb}$ was suspected unless $\mathrm{Tb}$ treatment had been initiated already. Nevertheless, 3 cases of $\mathrm{Tb}$ were observed in the 5 trials included in this review (Table 4), indicating that caution should prevail in patients receiving adalimumab for psoriasis even in the absence of signs for latent $\mathrm{Tb}$ prior to treatment initiation. Other severe infections observed in clinical trials assessing adalimumab for psoriasis included coccidioidomycosis $(n=1)^{48}$ and viral meningitis $(n=1) .{ }^{56-58}$ We did not identify any case reports 


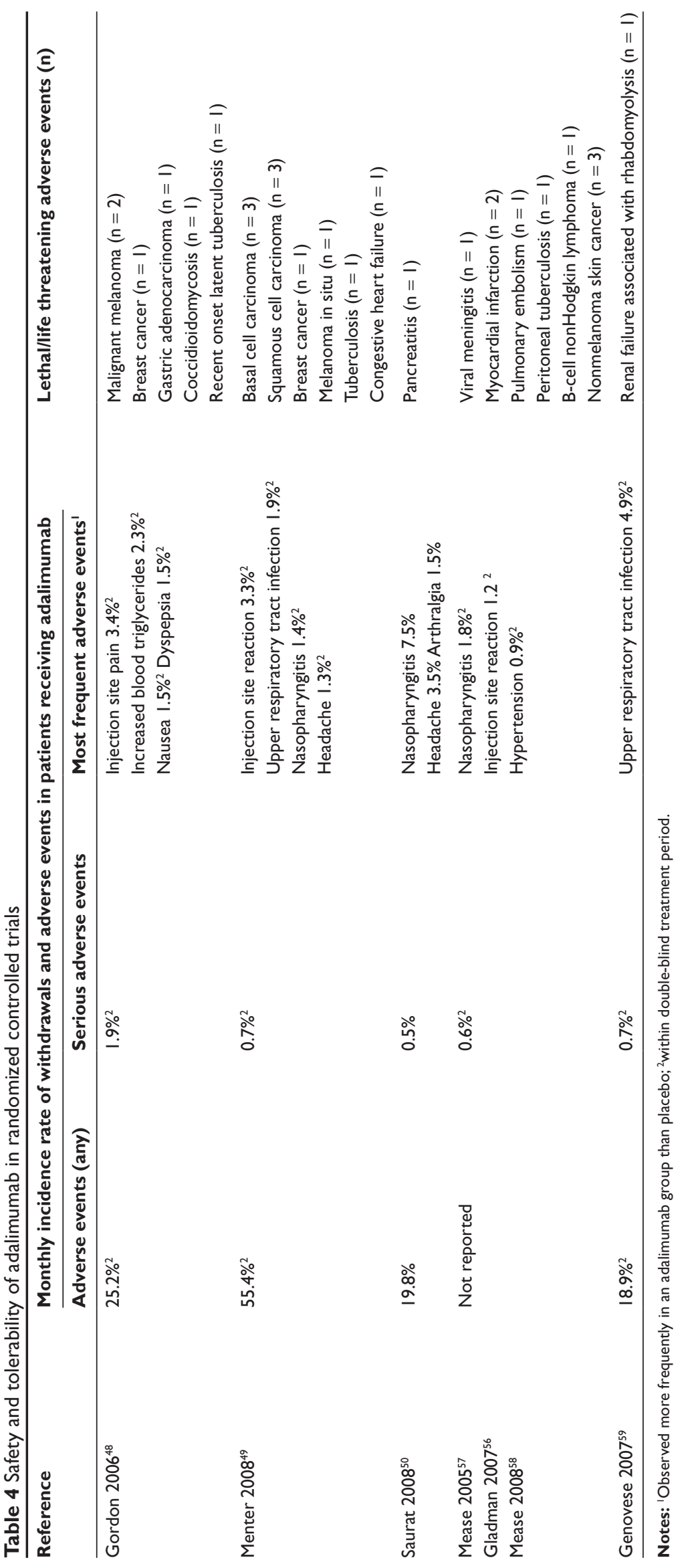


pointing at opportunistic infections in psoriasis patients treated with adalimumab. In patients receiving adalimumab for rheumatoid arthritis, however, a series of cases with Legionella pneumophila pneumonia has been reported recently. ${ }^{79}$

Table 4 summarizes the cases of malignant disease observed in patients treated with adalimumab in RCTs. For most tumor entities only single cases were observed, allowing no conclusions about the association with adalimumab. A total of 10 cases of nonmelanoma skin cancers and 3 cases of malignant melanoma were observed (Table 4). Fulchiero et al reported a case of late recurrence of locoregional metastatic melanoma shortly after the initiation of adalimumab for rheumatoid arthritis. ${ }^{73}$ Other cases of late recurrence of melanoma in psoriasis patients receiving anti-TNF $\alpha$ treatment have been reported (summary in ${ }^{73}$ ), so that patients with a history of melanoma have been not eligible in trials assessing adalimumab for psoriasis. ${ }^{48-50,57,59}$ Although the incidence of melanoma and nonmelanoma skin cancers in clinical trials is relatively high, it may still be explained by chance. Development of malignant melanoma and nonmelanoma skin cancer is a general concern for patients undergoing immunosuppressive therapy and/or phototherapy. ${ }^{95-99}$ Because patients receiving adalimumab were typically exposed to other immunosuppressants/ immune-modifying agents before, the role of adalimumab in the reported cases of skin cancer remains unclear. Until representative safety data are available, each patient's individual risk factor profile for nonmelanoma skin cancer and melanoma history should be carefully considered before a decision is taken on whether adalimumab (or any other $\mathrm{TNF} \alpha$ therapy) is appropriate.

Cases of demyelination, optic neuritis, and multiple sclerosis (MS) have not been observed in RCTs of adalimumab for plaque-type psoriasis or PsA (Table 4). We identified 2 case reports which document a possible association of adalimumab treatment with the development of demyelination ${ }^{71}$ and optic neuritis (Table 5)..$^{72}$ This association is plausible, because several reports of MS or associated symptoms have been reported under treatment with other anti-TNF $\alpha$ agents, particularly with etanercept, but also with infliximab. ${ }^{71,100}$ A placebo-controlled RCT evaluating the effect of lenercept, a recombinant TNF receptor p55 immunoglobulin fusion protein, in patients with MS showed an increase in exacerbations and more severe neurologic deficits in patients receiving lenercept compared to placebo. ${ }^{101}$ Prospective registries are necessary to clarify if and to what extend adalimumab is associated with an increased risk of central nervous demyelination. Until then, doctors should inform patients about this possible association, avoid the use of adalimumab in patients with a history of MS, and be aware of early signs of demyelinating disease such as weakness in the limb(s), paresthesia, visual blurring, and ataxia.

Deng et al reported 5 cases of interstitial granulomatous dermatitis developing in patients treated with TNF $\alpha$ antagonists including adalimumab (Table 5). ${ }^{78}$ Although it remains unclear from the reported data whether adalimumab caused this reaction or whether it was just a coincidence, interstitial granulomatous dermatitis should be considered in the differential diagnosis of annular lesions occurring in patients treated with adalimumab.

Numerous reports of the induction or worsening of psoriasis in patients treated with TNF $\alpha$ antagonists indicate that this is not a rare phenomenon. ${ }^{81,82}$ Collamer et al reviewed all cases of pustular psoriasis occurring under anti-TNF $\alpha$ therapy and concluded that new-onset psoriasis may occur any time after initiation anti-TNF $\alpha$ therapy and is often of an uncommon morphology or localization. ${ }^{102} \mathrm{We}$ identified 2 cases of new onset generalized pustular psoriasis in patients receiving adalimumab for $\mathrm{PsA}^{80}$ and pustular psoriasis of the scalp, ${ }^{81}$ respectively. The etiology of this adverse reaction is not completely understood yet. It has been suggested that this paradoxical response appears to involve a disruption in cytokine balance following TNF $\alpha$ inhibition, resulting in the upregulation of plasmacytoid dendritic cells and the subsequent production of unopposed interferon-alpha, following a triggering event in predisposed individuals. ${ }^{102}$ Notably, adalimumab has not only been reported to cause pustular psoriasis, but also to be effective in the treatment of recalcitrant generalized pustular psoriasis in adults and adolescents. ${ }^{83,84}$

Adalimumab-induced psoriasis does not necessarily require therapy cessation. Particularly in patients with severe PsA and good clinical response to adalimumab, we recommend aggressive treatment of the skin lesions and discontinuation of adalimumab only in cases unresponsive to topical antipsoriatic treatment.

In patients treated with adalimumab for rheumatoid arthritis or psoriasis several cutaneous adverse reactions other than new onset psoriasis may occur, including subacute cutaneous lupus erythematosus, vitiligo, urticaria, eczema, dermatitis herpetiformis, perivascular neutrophilic dermatitis, tinea, alopecia areata, and pityriasis rosea. ${ }^{74,75,77,103-106}$ Most of these reactions were mild and did not require discontinuation of treatment. The development of these conditions shortly 


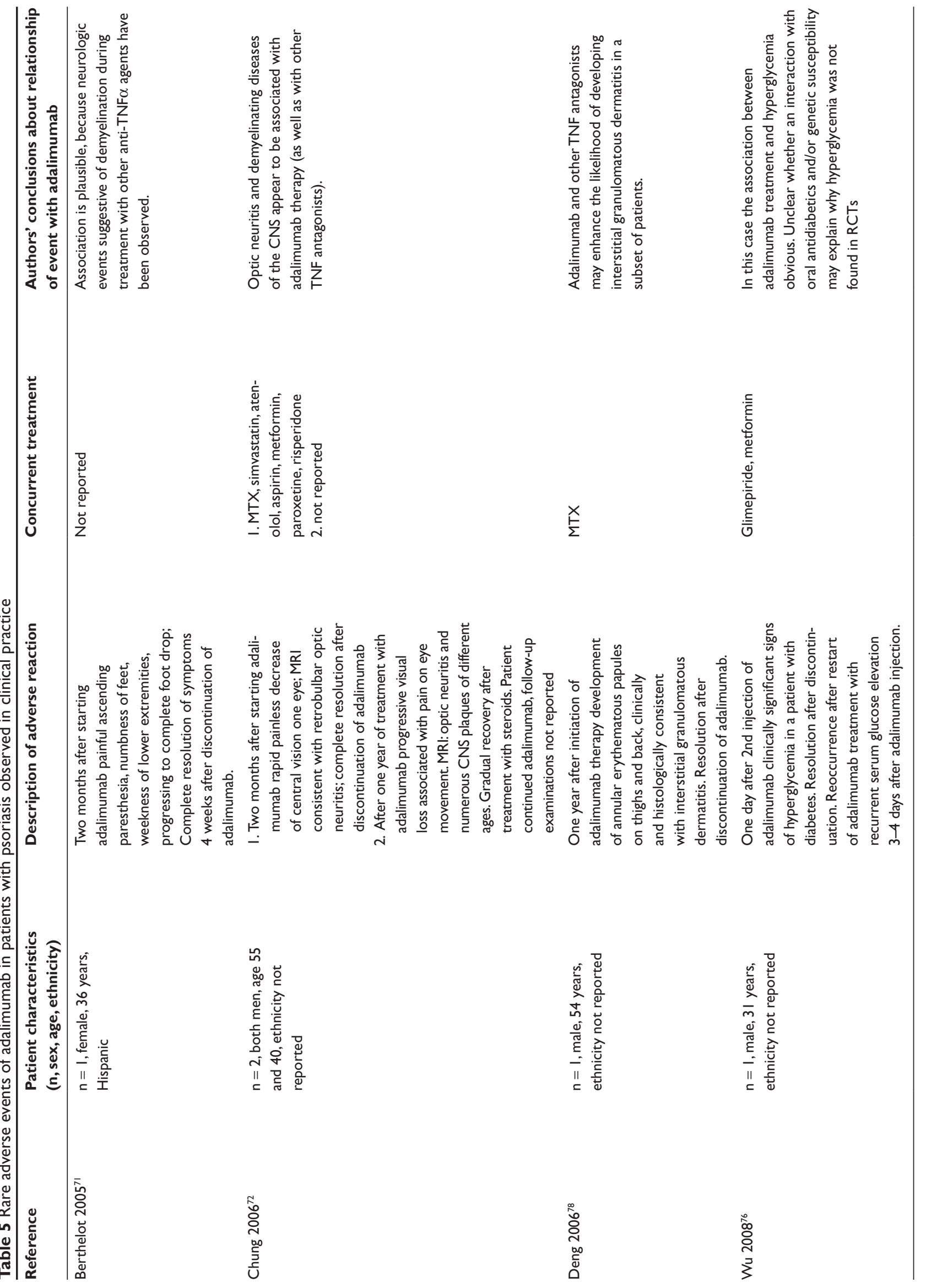


after the initiation of adalimumab treatment and the improvement/clearance after discontinuation of adalimumab suggests a causal relationship. However, most of these conditions are quite frequent in the general population, so that some of these reactions may have been coincidences rather than associations with adalimumab. Prospective registries are needed for further clarification.

Adalimumab is often prescribed to women of reproductive age, raising questions about its effect on pregnancy. The molecular structure of adalimumab permits little placental transfer during the first trimester, but placental transfer cannot be excluded during the second and third trimesters of pregnancy. ${ }^{107}$ In animal studies, no evidence of embryotoxicity or teratogenicity has been observed. ${ }^{70}$ Human experience is still extremely limited, particularly in patients with psoriasis. One patient with rheumatoid arthritis became pregnant after receiving a single dose of adalimumab. She delivered a healthy infant at 32 weeks with no neonatal abnormalities and normal growth and development. ${ }^{70}$ In patients becoming pregnant while receiving anti-TNF $\alpha$ therapy the following perinatal complications were observed: prematurity, neonatal jaundice, neonatal urinary Escherichia coli infection, and adrenal congenital hyperplasia of probable hereditary origin. ${ }^{70}$ Therefore, caution should be taken when adalimumab or other anti-TNF agents are used in women with childbearing potential. Any cases of pregnancy under adalimumab should be collected in registries to be able to give evidence-based recommendations for consulting pregnant women under adalimumab therapy in the future.

Data on the safety of adalimumab and other TNF $\alpha$ antagonists in HIV-positive individuals and in patients with viral hepatitis are limited to few case series, which did not detect any significant clinical adverse effects attributed to anti-TNF treatment. ${ }^{108,109}$ However, systematic, studies and/ or prospective registries addressing the issue of safety of monoclonal antibodies targeted against TNF $\alpha$ in patients with chronic hepatitis and in HIV-positive individuals will be needed to properly assess the risks and benefits of anti-TNF treatment in these patients usually excluded from clinical trials.

\section{Efficiency/cost-effectiveness of adalimumab for psoriasis}

We identified 2 studies assessing the cost-effectiveness of adalimumab in plaque-type psoriasis. Sizto et al compared the long-term cost-effectiveness of biologic and nonbiologic treatment options for moderate to severe plaque-type psoriasis. ${ }^{68}$ Within the group of biologics adalimumab was most cost-effective, followed by etanercept. Despite lower efficacy, methotrexate and cyclosporine were more cost-effective than adalimumab due to the lower direct cost of these prebiologic systemic agents. ${ }^{68}$ Because data on the long-term effectiveness of the different agents approved for psoriasis are sparse, this analysis is limited by a number of assumptions based on expert opinions rather than published data. According to another health-economic evaluation of biologic agents for psoriasis Nelson et al assessed infliximab to be most cost-effective in the short-term biologic treatment of plaque-type psoriasis, followed by adalimumab. ${ }^{67}$ It has been reported, however, that the cost-effectiveness of biologics are observed to converge over the first year of treatment, so that extrapolating the results of the study by Nelson et al to longer treatment periods may not be accurate. ${ }^{67,69}$

\section{Comment}

In recent years, the development and approval of injectable biologic agents has revolutionized the management of moderate-to-severe plaque-type psoriasis and PsA. In contrast to $\mathrm{T}$ cell inhibitors, $\mathrm{TNF} \alpha$ antagonists are providing concomitant benefits for the skin and joints.

Among these, adalimumab is the first fully human monoclonal antibody against TNF $\alpha$.

Studies indicate that it is highly efficacious both in moderate-to-severe plaque type psoriasis and in severe PsA with significant improvements within 4 weeks. ${ }^{48-61}$ A recent meta-analysis indicated that adalimumab is superior to cyclosporine, efalizumab, and etanercept in the treatment of chronic plaque-type psoriasis. ${ }^{110}$ Based on published trials, infliximab leads to higher response rates than adalimumab within 12 to 16 weeks of treatment. However, a loss of efficacy over time has been observed in patients receiving infliximab, but does not appear to be an issue for adalimumab based on published data. ${ }^{110}$ One head-to-head study suggested that adalimumab is at least similarly efficacious and possibly more efficacious than MTX in the short-term treatment of moderate-to-severe plaque-type psoriasis. ${ }^{50,55}$

Managed care organizations are currently debating the appropriate use of biologic drugs for psoriasis. ${ }^{11,112}$ Given the widespread patient dissatisfaction with conventional treatments, the demand of treatment alternatives is high. ${ }^{10}$ In all published RCTs clinical improvements translated into increased wellbeing, improved health-related quality of life, and improvements in other patient-reported outcomes. Future studies should investigate the comparative efficacy of adalimumab and other biologic and prebiologic agents.

Adalimumab treatment may be particularly beneficial for psoriasis patients who have failed multiple other biologic and 
nonbiologic systemic agents: effectiveness studies suggested that adalimumab is highly effective in the subset of patients not responding to other conventional of biologic treatments approved for psoriasis and that adalimumab therapy is more likely to be continued than treatment with other anti-TNF $\alpha$ agents. Concomitant MTX therapy should be considered particularly in cases of severe PsA.

Pharmaco-genetic investigations suggested that the response to anti-TNF $\alpha$ agents including adalimumab is modified by polymorphisms in the TNF $\alpha$-gene and Fc $\gamma$-receptor gene. ${ }^{65,66}$ These studies are very promising and may help to better identify the subgroup of psoriasis patients with psoriasis benefiting most from adalimumab therapy. Prior to the application of gene-polymorphism screening in clinical practice, however, replications of these promising findings and similar studies in patients with plaque-type psoriasis are warranted.

Economic evaluations indicated that adalimumab is one of the biologics with a more favorable cost-benefit profile. ${ }^{67-69}$ One study suggested that adalimumab may be less efficient than methotrexate and cyclosporine. ${ }^{68}$ More studies with a longer time horizon and a more rigorous methodology considering also indirect costs are needed to better understand economic implications of a broader and possibly earlier use of biologics.

Despite significant theoretical risks, the safety profiles of TNF $\alpha$ antagonists such as adalimumab are remarkably good. Burmester et al summarized data on the safety of adalimumab from 19,041 patients exposed to adalimumab in 36 clinical trials in psoriasis, PsA, rheumatoid arthritis, ankylosing spondylitis, and Crohn's disease and concluded that the overall malignancy rates and the all-cause mortality rates for adalimumab-treated patients were as expected for the general population. ${ }^{113}$

Prior to initiation of adalimumab therapy patients should be evaluated for active/latent $\mathrm{Tb}$, serious infections, and other contraindications.

Recently established registries are expected to yield more data on the safety and effectiveness of adalimumab in clinical practice soon.

\section{Disclosures}

JS: None.

GW: Prof. Wozel has been an investigator in clinical trials and served as a consultant for Abbott, Biogen-Idec, Centocor, Essex, Serono, and Wyeth.

\section{References}

1. Griffiths CE, Barker JN. Pathogenesis and clinical features of psoriasis. Lancet. 2007;370:263-271.

2. Naldi L. Epidemiology of psoriasis. Curr Drug Targets Inflamm Allergy. 2004;3:121-128.
3. de Korte J, Sprangers MA, Mombers FM, et al. Quality of life in patients with psoriasis: a systematic literature review. J Investig Dermatol Symp Proc. 2004;9:140-147.

4. Mallbris L, Akre O, Granath F, et al. Increased risk for cardiovascular mortality in psoriasis inpatients but not in outpatients. Eur J Epidemiol. 2004; 19:225-230.

5. Kremers HM, McEvoy MT, Dann FJ, et al. Heart disease in psoriasis. J Am Acad Dermatol. 2007;57:347-354.

6. Gelfand JM, Troxel AB, Lewis JD, et al. The risk of mortality in patients with psoriasis: results from a population-based study. Arch Dermatol. 2007;143:1493-1499.

7. Gelfand JM, Neimann AL, Shin DB, et al. Risk of myocardial infarction in patients with psoriasis. JAMA. 2006;296:1735-1741.

8. Schmitt JM, Ford DE. Role of depression in quality of life for patients with psoriasis. Dermatology. 2007;215:17-27.

9. Gupta MA, Schork NJ, Gupta AK, et al. Suicidal ideation in psoriasis. Int J Dermatol. 1993;32:188-190.

10. Stern RS, Nijsten T, Feldman SR, et al. Psoriasis is common, carries a substantial burden even when not extensive, and is associated with widespread treatment dissatisfaction. J Investig Dermatol Symp Proc. 2004;9:136-139.

11. Javitz HS, Ward MM, Farber E, et al. The direct cost of care for psoriasis and psoriatic arthritis in the United States. J Am Acad Dermatol. 2002;46:850-860.

12. Schmitt J, Ford DE. Work Limitations and Productivity Loss are Associated with Health-related Quality of Life but not with Clinical Severity in Patients with Psoriasis. Dermatology. 2006;213:110.

13. Menter A, Griffiths CE. Current and future management of psoriasis. Lancet. 2007;370:272-284.

14. Nast A, Kopp IB, Augustin M, et al. [S3-Guidelines for the therapy of psoriasis vulgaris]. J Dtsch Dermatol Ges. 2006;4 Suppl 2:S1-126.

15. British Association of Dermatologists. Psoriasis Guideline 2006. Available at http://www.library.nhs.uk/guidelinesfinder/ViewResource. aspx?resID=125323. 2006. British Association of Dermatologists. $31=1-2008$.

16. Mosmann TR, Cherwinski H, Bond MW, et al. Two types of murine helper T cell clone. I. Definition according to profiles of lymphokine activities and secreted proteins. 1986. J Immunol. 2005;175:5-14.

17. Nickoloff BJ. Cracking the cytokine code in psoriasis. Nat Med. 2007;13:242-244.

18. Papp KA, Langley RG, Lebwohl M, et al. Efficacy and safety of ustekinumab, a human interleukin-12/23 monoclonal antibody, in patients with psoriasis: 52-week results from a randomised, double-blind, placebo-controlled trial (PHOENIX 2). Lancet. 2008;371:1675-1684.

19. Leonardi CL, Kimball AB, Papp KA, et al. Efficacy and safety of ustekinumab, a human interleukin-12/23 monoclonal antibody, in patients with psoriasis: 76-week results from a randomised, double-blind, placebo-controlled trial (PHOENIX 1). Lancet. 2008;371:1665-1674.

20. Kimball AB, Gordon KB, Langley RG, et al. Safety and efficacy of ABT-874, a fully human interleukin 12/23 monoclonal antibody, in the treatment of moderate to severe chronic plaque psoriasis: results of a randomized, placebo-controlled, phase 2 trial. Arch Dermatol. 2008;144:200-207.

21. EMEA. European Medicines Agency recommends suspension of the marketing authorisation of Raptiva (efalizumab). 19 Feb 2009. London, UK, EMEA.

22. Schottelius AJ, Moldawer LL, Dinarello CA, et al. Biology of tumor necrosis factor-alpha-implications for psoriasis. Exp Dermatol. 2004;13:193-222.

23. Wakefield PE, James WD, Samlaska CP, et al. Tumor necrosis factor. J Am Acad Dermatol. 1991;24:675-685.

24. Davenport CM, McAdams HA, Kou J, et al. Inhibition of pro-inflammatory cytokine generation by CTLA4-Ig in the skin and colon of mice adoptively transplanted with CD45RBhi CD4+ T cells correlates with suppression of psoriasis and colitis. Int Immunopharmacol. 2002;2:653-672.

25. Nickoloff BJ, Karabin GD, Barker JN, et al. Cellular localization of interleukin-8 and its inducer, tumor necrosis factor-alpha in psoriasis. Am J Pathol. 1991;138:129-140. 
26. Kristensen M, Chu CQ, Eedy DJ, et al. Localization of tumour necrosis factor-alpha (TNF-alpha) and its receptors in normal and psoriatic skin: epidermal cells express the $55-\mathrm{kD}$ but not the $75-\mathrm{kD}$ TNF receptor. Clin Exp Immunol. 1993;94:354-362.

27. Ettehadi P, Greaves MW, Wallach D, et al. Elevated tumour necrosis factor-alpha (TNF-alpha) biological activity in psoriatic skin lesions. Clin Exp Immunol. 1994;96:146-151.

28. Bonifati C, Carducci M, Cordiali FP, et al. Correlated increases of tumour necrosis factor-alpha, interleukin- 6 and granulocyte monocyte-colony stimulating factor levels in suction blister fluids and sera of psoriatic patients-relationships with disease severity. Clin Exp Dermatol. 1994;19:383-387.

29. Mussi A, Bonifati C, Carducci M, et al. Serum TNF-alpha levels correlate with disease severity and are reduced by effective therapy in plaque-type psoriasis. J Biol Regul Homeost Agents. 1997;11:115-118.

30. Uyemura K, Yamamura M, Fivenson DF, et al. The cytokine network in lesional and lesion-free psoriatic skin is characterized by a T-helper type 1 cell-mediated response. J Invest Dermatol. 1993;101:701-705.

31. Partsch G, Wagner E, Leeb BF, et al. Upregulation of cytokine receptors sTNF-R55, sTNF-R75, and sIL-2R in psoriatic arthritis synovial fluid. J Rheumatol. 1998;25:105-110.

32. Ritchlin C, Haas-Smith SA, Hicks D, et al. Patterns of cytokine production in psoriatic synovium. J Rheumatol. 1998;25:1544-1552.

33. Hohler T, Kruger A, Schneider PM, et al. A TNF-alpha promoter polymorphism is associated with juvenile onset psoriasis and psoriatic arthritis. J Invest Dermatol. 1997;109:562-565.

34. Beyaert R, De Potter C, Vanhaesebroeck B, et al. Induction of inflammatory cell infiltration and necrosis in normal mouse skin by the combined treatment of tumor necrosis factor and lithium chloride. Am J Pathol. 1991;138:727-739.

35. Palladino MA, Bahjat FR, Theodorakis EA, et al. Anti-TNF-alpha therapies: the next generation. Nat Rev Drug Discov. 2003;2:736-746.

36. Tracey D, Klareskog L, Sasso EH, et al. Tumor necrosis factor antagonist mechanisms of action: a comprehensive review. Pharmacol Ther. 2008;117:244-279.

37. Sabat R, Philipp S, Hoflich C, et al. Immunopathogenesis of psoriasis. Exp Dermatol. 2007;16:779-798.

38. Boehncke WH, Prinz J, Gottlieb AB. Biologic therapies for psoriasis. A systematic review. J Rheumatol. 2006;33:1447-1451.

39. Kempeni J. Preliminary results of early clinical trials with the fully human anti-TNFalpha monoclonal antibody D2E7. Ann Rheum Dis. 1999;58 Suppl 1:I70-I72.

40. EMEA. SCIENTIFIC DISCUSSION, Humira (Adalimumab). Available at http://www.emea.europa.eu/humandocs/PDFs/EPAR/ humira/400803en6.pdf, accessed March 24, 2009. 2004.

41. FDA. Package insert Humira (adalimumab), U.S. Govt. Lic. No. 0043, Abbott Laboratories. Available at http://www.fda.gov/Cder/foi/ label/2002/adalabb123102LB.htm, accessed March 24, 2009. 2002.

42. Weisman MH, Moreland LW, Furst DE, et al. Efficacy, pharmacokinetic, and safety assessment of adalimumab, a fully human anti-tumor necrosis factor-alpha monoclonal antibody, in adults with rheumatoid arthritis receiving concomitant methotrexate: a pilot study. Clin Ther. 2003;25:1700-1721.

43. DeRuiter J, Riley TN. How can adalimumab (Humira) be used to treat rheumatoid arthritis? US Pharmacist. [serial online] 2003;28(5).

44. Santora LC, Kaymakcalan Z, Sakorafas P, et al. Characterization of noncovalent complexes of recombinant human monoclonal antibody and antigen using cation exchange, size exclusion chromatography, and BIAcore. Anal Biochem. 2001;299:119-129.

45. Tjioe M, Gerritsen MJ, Den Broeder AA, et al. Adalimumab, a fully human anti-TNF-alpha monoclonal antibody, treatment does not influence experimental UV response in the skin of rheumatoid arthritis patients. Exp Dermatol. 2003;12:460-465.

46. Fredriksson T, Pettersson U. Severe psoriasis--oral therapy with a new retinoid. Dermatologica. 1978;157:238-244.

47. Felson DT, Anderson JJ, Boers M, et al. American College of Rheumatology. Preliminary definition of improvement in rheumatoid arthritis. Arthritis Rheum. 1995;38:727-735.
48. Gordon KB, Langley RG, Leonardi C, et al. Clinical response to adalimumab treatment in patients with moderate to severe psoriasis: double-blind, randomized controlled trial and open-label extension study. J Am Acad Dermatol. 2006;55:598-606.

49. Menter A, Tyring SK, Gordon K, et al. Adalimumab therapy for moderate to severe psoriasis: A randomized, controlled phase III trial. J Am Acad Dermatol. 2008;58:106-115.

50. Saurat JH, Stingl G, Dubertret L, et al. Efficacy and safety results from the randomized controlled comparative study of adalimumab vs. methotrexate vs. placebo in patients with psoriasis (CHAMPION). $\mathrm{Br} J$ Dermatol. 2008;158:558-566.

51. Revicki DA, Menter A, Feldman S, et al. Adalimumab improves health-related quality of life in patients with moderate to severe plaque psoriasis compared with the United States general population norms: results from a randomized, controlled Phase III study. Health Qual Life Outcomes. 2008;6:75

52. Revicki D, Willian MK, Saurat JH, et al. Impact of adalimumab treatment on health-related quality of life and other patient-reported outcomes: results from a 16-week randomized controlled trial in patients with moderate to severe plaque psoriasis. Br J Dermatol. 2008;158:549-557.

53. Revicki DA, Willian MK, Menter A, et al. Impact of adalimumab treatment on patient-reported outcomes: results from a Phase III clinical trial in patients with moderate to severe plaque psoriasis. J Dermatolog Treat. 2007;18:341-350.

54. Shikiar R, Heffernan M, Langley RG, et al. Adalimumab treatment is associated with improvement in health-related quality of life in psoriasis: patient-reported outcomes from a phase II randomized controlled trial. J Dermatolog Treat. 2007;18:25-31.

55. Nijsten T, Spuls PI. Adalimumab may be better or no worse than methotrexate in the treatment of psoriasis. Br J Dermatol. 2008;159:257-258.

56. Gladman DD, Mease PJ, Ritchlin CT, et al. Adalimumab for longterm treatment of psoriatic arthritis: forty-eight week data from the adalimumab effectiveness in psoriatic arthritis trial. Arthritis Rheum. 2007;56:476-488

57. Mease PJ, Gladman DD, Ritchlin CT, et al. Adalimumab for the treatment of patients with moderately to severely active psoriatic arthritis: results of a double-blind, randomized, placebo-controlled trial. Arthritis Rheum. 2005;52:3279-3289.

58. Mease PJ, Ory P, Sharp JT, et al. Adalimumab for long-term treatment of psoriatic arthritis: two-year data from the Adalimumab Effectiveness in Psoriatic Arthritis Trial (ADEPT). Ann Rheum Dis. 2009;69:702-709.

59. Genovese MC, Mease PJ, Thomson GT, et al. Safety and efficacy of adalimumab in treatment of patients with psoriatic arthritis who had failed disease modifying antirheumatic drug therapy. J Rheumatol. 2007;34:1040-1050.

60. Revicki DA, Willian MK, Menter A, et al. Relationship between clinical response to therapy and health-related quality of life outcomes in patients with moderate to severe plaque psoriasis. Dermatology. 2008;216:260-270.

61. Gladman DD, Mease PJ, Cifaldi MA, et al. Adalimumab improves joint-related and skin-related functional impairment in patients with psoriatic arthritis: patient-reported outcomes of the Adalimumab Effectiveness in Psoriatic Arthritis Trial. Ann Rheum Dis. 2007;66: 163-168.

62. Papoutsaki M, Chimenti MS, Costanzo A, et al. Adalimumab for severe psoriasis and psoriatic arthritis: an open-label study in 30 patients previously treated with other biologics. J Am Acad Dermatol. 2007;57: 269-275.

63. Heiberg MS, Koldingsnes W, Mikkelsen K, et al. The comparative one-year performance of anti-tumor necrosis factor alpha drugs in patients with rheumatoid arthritis, psoriatic arthritis, and ankylosing spondylitis: results from a longitudinal, observational, multicenter study. Arthritis Rheum. 2008;59:234-240.

64. Van L, Modi SV, Yang DJ, et al. Sustained efficacy and safety of adalimumab in psoriasis treatment: a retrospective study of 49 patients with and without a history of TNF-alpha antagonist treatment. Arch Dermatol. 2008;144:804-806. 
65. Seitz M, Wirthmuller U, Moller B, et al. The -308 tumour necrosis factor-alpha gene polymorphism predicts therapeutic response to TNFalpha-blockers in rheumatoid arthritis and spondyloarthritis patients. Rheumatology (Oxford). 2007;46:93-96.

66. Tutuncu Z, Kavanaugh A, Zvaifler N, et al. Fcgamma receptor type IIIA polymorphisms influence treatment outcomes in patients with inflammatory arthritis treated with tumor necrosis factor alpha-blocking agents. Arthritis Rheum. 2005;52:2693-2696.

67. Nelson AA, Pearce DJ, Fleischer AB, Jr, et al. Cost-effectiveness of biologic treatments for psoriasis based on subjective and objective efficacy measures assessed over a 12 -week treatment period. $\mathrm{J} \mathrm{Am}$ Acad Dermatol. 2008;58:125-135.

68. Sizto S, Bansback N, Feldman SR, et al. Economic evaluation of systemic therapies for moderate to severe psoriasis. Br J Dermatol. 2008; Dec 13 [Epub ahead of print].

69. Goldberg LD. The convergence of psoriasis treatment cost-effectiveness over time: "real world" considerations in economic modeling. J Am Acad Dermatol. 2008;58:1073-1075.

70. Roux CH, Brocq O, Breuil V, et al. Pregnancy in rheumatology patients exposed to anti-tumour necrosis factor (TNF)-alpha therapy. Rheumatology (Oxford). 2007;46:695-698.

71. Berthelot CN, George SJ, Hsu S. Distal lower extremity paresthesia and foot drop developing during adalimumab therapy. J Am Acad Dermatol. 2005;53:S260-S262.

72. Chung JH, Van Stavern GP, Frohman LP, et al. Adalimumab-associated optic neuritis. J Neurol Sci. 2006;244:133-136.

73. Fulchiero GJ Jr, Salvaggio H, Drabick JJ, et al. Eruptive latent metastatic melanomas after initiation of antitumor necrosis factor therapies. $J \mathrm{Am}$ Acad Dermatol. 2007;56:S65-S67.

74. George SJ, Anderson HL, Hsu S. Adalimumab-induced urticaria Dermatol Online J. 2006;12:4.

75. Smith DI, Heffernan MP. Vitiligo after the resolution of psoriatic plaques during treatment with adalimumab. J Am Acad Dermatol. 2008;58:S50-S52.

76. Wu JJ, Tsai TF. Recurrent hyperglycemia during adalimumab treatment in a patient with psoriasis. Arch Dermatol. 2008;144:1403-1404.

77. Sheth N, Greenblatt D, Patel S, et al. Adalimumab-induced cutaneous lupus. Clin Exp Dermatol. 2007;32:593-594.

78. Deng A, Harvey V, Sina B, et al. Interstitial granulomatous dermatitis associated with the use of tumor necrosis factor alpha inhibitors. Arch Dermatol. 2006;142:198-202.

79. Tubach F, Ravaud P, Salmon-Ceron D, et al. Emergence of Legionella pneumophila pneumonia in patients receiving tumor necrosis factor-alpha antagonists. Clin Infect Dis. 2006;43:e95-e100.

80. Dalmau J, Roe E, Corella F, et al. Acute generalized skin eruption due to adalimumab: report of two cases. J Eur Acad Dermatol Venereol. 2007;21:1105-1106.

81. Wollina U, Hansel G, Koch A, et al. Tumor necrosis factor-alpha inhibitor-induced psoriasis or psoriasiform exanthemata: first 120 cases from the literature including a series of six new patients. Am J Clin Dermatol. 2008;9:1-14.

82. Papadavid E, Gazi S, Dalamaga M, et al. Palmoplantar and scalp psoriasis occurring during anti-tumour necrosis factor-alpha therapy: a case series of four patients and guidelines for management. J Eur Acad Dermatol Venereol. 2008;22:380-382.

83. Zangrilli A, Papoutsaki M, Talamonti M, et al. Long-term efficacy of adalimumab in generalized pustular psoriasis. $J$ Dermatolog Treat. 2008;19:185-187.

84. Callen JP, Jackson JH. Adalimumab effectively controlled recalcitrant generalized pustular psoriasis in an adolescent. J Dermatolog Treat. 2005; $16: 350-352$

85. Stinco G, Piccirillo F, Patrone P. Hypertriglyceridaemia during treatment with adalimumab in psoriatic arthritis. $\mathrm{Br} J$ Dermatol. 2007; 157:1273-1274.

86. Ware JE Jr, Sherbourne CD. The MOS 36-item short-form health survey (SF-36). I. Conceptual framework and item selection. Med Care. 1992;30:473-483.
87. McHorney CA, Ware JE Jr, Raczek AE. The MOS 36-Item Short-Form Health Survey (SF-36): II. Psychometric and clinical tests of validity in measuring physical and mental health constructs. Med Care. 1993;31:247-263.

88. Finlay AY, Khan GK. Dermatology Life Quality Index (DLQI) - a simple practical measure for routine clinical use. Clin Exp Dermatol. 1994;19:210-216.

89. Brazier J, Jones N, Kind P. Testing the validity of the Euroqol and comparing it with the SF-36 health survey questionnaire. Qual Life Res. 1993;2:169-180.

90. Patel T, Gordon KB. Adalimumab: efficacy and safety in psoriasis and rheumatoid arthritis. Dermatol Ther. 2004;17:427-431.

91. Mossner R, Schon MP, Reich K. Tumor necrosis factor antagonists in the therapy of psoriasis. Clin Dermatol. 2008;26:486-502.

92. Richeldi L. An update on the diagnosis of tuberculosis infection. Am J Respir Crit Care Med. 2006;174:736-742.

93. Lalvani A. Diagnosing tuberculosis infection in the 21 st century: new tools to tackle an old enemy. Chest. 2007;131:1898-1906.

94. Doherty SD, Van Voorhees A, Lebwohl MG, et al. National Psoriasis Foundation consensus statement on screening for latent tuberculosis infection in patients with psoriasis treated with systemic and biologic agents. J Am Acad Dermatol. 2008;59:209-217.

95. Bouwes Bavinck JN, Hardie DR, Green A, et al. The risk of skin cancer in renal transplant recipients in Queensland, Australia. A follow-up study. Transplantation. 1996;61:715-721.

96. Jensen P, Hansen S, Moller B, et al. Skin cancer in kidney and heart transplant recipients and different long-term immunosuppressive therapy regimens. J Am Acad Dermatol. 1999;40:177-186.

97. Euvrard S, Kanitakis J, Claudy A. Skin cancers after organ transplantation. $N$ Engl J Med. 2003;348:1681-1691.

98. Saladi RN, Persaud AN. The causes of skin cancer: a comprehensive review. Drugs Today (Barc). 2005;41:37-53.

99. Marcil I, Stern RS. Squamous-cell cancer of the skin in patients given PUVA and ciclosporin: nested cohort crossover study. Lancet. 2001;358:1042-1045.

100. Mohan N, Edwards ET, Cupps TR, et al. Demyelination occurring during anti-tumor necrosis factor alpha therapy for inflammatory arthritides. Arthritis Rheum. 2001;44:2862-2869.

101. TNF neutralization in MS: results of a randomized, placebo-controlled multicenter study. The Lenercept Multiple Sclerosis Study Group and The University of British Columbia MS/MRI Analysis Group. Neurology. 1999;53:457-465.

102. Collamer AN, Guerrero KT, Henning JS, et al. Psoriatic skin lesions induced by tumor necrosis factor antagonist therapy: a literature review and potential mechanisms of action. Arthritis Rheum. 2008;59:996-1001.

103. Lee HH, Song IH, Friedrich M, et al. Cutaneous side-effects in patients with rheumatic diseases during application of tumour necrosis factor-alpha antagonists. Br J Dermatol. 2007;156:486-491.

104. Scheinfeld N. Adalimumab: a review of side effects. Expert Opin Drug Saf. 2005;4:637-641.

105. Kirshen C, Kanigsberg N. Alopecia areata following adalimumab. J Cutan Med Surg. 2009;13:48-50.

106. Garcia BN, Lee HH, Worm M, et al. Development of alopecia areata universalis in a patient receiving adalimumab. Arch Dermatol. 2006;142:1654-1655.

107. Simister NE. Placental transport of immunoglobulin G. Vaccine. 2003;21:3365-3369.

108. Cepeda EJ, Williams FM, Ishimori ML, et al. The use of anti-tumour necrosis factor therapy in HIV-positive individuals with rheumatic disease. Ann Rheum Dis. 2008;67:710-712.

109. Aslanidis S, Vassiliadis T, Pyrpasopoulou A, et al. Inhibition of TNFalpha does not induce viral reactivation in patients with chronic hepatitis C infection: two cases. Clin Rheumatol. 2007;26:261-264.

110. Schmitt J, Zhang Z, Wozel G, et al. Efficacy and tolerability of biologic and nonbiologic systemic treatments for moderate-to-severe psoriasis: meta-analysis of randomized controlled trials. Br J Dermatol. 2008;159:513-526. 
111. Rich SJ. Considerations for assessing the cost of biologic agents in the treatment of psoriasis. J Manag Care Pharm. 2004;10:S38-S41.

112. Stein KR, Pearce DJ, Feldman SR. The impact of biologics on the quality of life of psoriasis patients and the economics of psoriasis care. Semin Cutan Med Surg. 2005;24:52-57.
113. Burmester GR, Mease PJ, Dijkmans BA, et al. Adalimumab safety and mortality rates from global clinical trials of six immune-mediated inflammatory diseases. Ann Rheum Dis. 2009; Jan 15 [Epub ahead of print].

\section{Publish your work in this journal}

Biologics: Targets \& Therapy is an international, peer-reviewed journal focusing on the patho-physiological rationale for and clinical application of Biologic agents in the management of autoimmune diseases, cancers or other pathologies where a molecular target can be identified. This journal is indexed on PubMed Central, CAS,
EMBase, Scopus and the Elsevier Bibliographic databases. The manuscript management system is completely online and includes a very quick and fair peer-review system, which is all easy to use. Visit http://www.dovepress.com/testimonials.php to read real quotes from published authors. 